\title{
Nonlinear Coupling in the Human Motor System
}

\author{
Chun-Chuan Chen, ${ }^{1,3}$ James M. Kilner, ${ }^{1}$ Karl J. Friston, ${ }^{1}$ Stefan J. Kiebel, ${ }^{1,4}$ Rohit K. Jolly, ${ }^{1}$ and Nick S. Ward ${ }^{2}$ \\ ${ }^{1}$ Wellcome Trust Centre for Neuroimaging and ${ }^{2}$ Sobell Department of Motor Neuroscience and Movement Disorders, Institute of Neurology, University \\ College London, WC1N 3BG London, United Kingdom, ${ }^{3}$ Graduate Institute of Biomedical Engineering, National Central University, Jhongli City, Taoyuan \\ County, 32001, Taiwan, and ${ }^{4}$ Max Planck Institute for Human Cognitive and Brain Sciences, 04303 Leipzig, Germany
}

The synchronous discharge of neuronal assemblies is thought to facilitate communication between areas within distributed networks in the human brain. This oscillatory activity is especially interesting, given the pathological modulation of specific frequencies in diseases affecting the motor system. Many studies investigating oscillatory activity have focused on same frequency, or linear, coupling between areas of a network. In this study, our aim was to establish a functional architecture in the human motor system responsible for induced responses as measured in normal subjects with magnetoencephalography. Specifically, we looked for evidence for additional nonlinear (between-frequency) coupling among neuronal sources and, in particular, whether nonlinearities were found predominantly in connections within areas (intrinsic), between areas (extrinsic) or both. We modeled the event-related modulation of spectral responses during a simple hand-grip using dynamic casual modeling. We compared models with and without nonlinear connections under conditions of symmetric and asymmetric interhemispheric connectivity. Bayesian model comparison suggested that the task-dependent motor network was asymmetric during right hand movements. Furthermore, it revealed very strong evidence for nonlinear coupling between sources in this distributed network, but interactions among frequencies within a source appeared linear in nature. Our results provide empirical evidence for nonlinear coupling among distributed neuronal sources in the motor system and that these play an important role in modulating spectral responses under normal conditions.

\section{Introduction}

Oscillatory activity is a ubiquitous feature of neuronal dynamics. It has been suggested that the synchronous discharge of neuronal assemblies might provide a mechanism to couple distributed processing over different neuronal populations (Singer and Gray, 1995; Kahana et al., 1999; Rodriguez et al., 1999). The central idea is that oscillations facilitate integration both within functionally segregated brain areas and between areas engaged by the same task. Many studies investigating oscillatory activity have focused on linear coupling between nodes of a network at the same frequency (Andrew and Pfurtscheller, 1996; Gerloff et al., 1998; Gross et al., 2001; Serrien et al., 2005). More recently, evidence has emerged that suggests nonlinear coupling among different frequencies may play an equally important role in interareal communication (TallonBaudry and Bertrand, 1999; Varela et al., 2001; Breakspear, 2002; Jensen and Colgin, 2007; Chen et al., 2009). In this work, we use magnetoencephalography (MEG) and a recently validated method (dynamic causal modeling for induced responses; DCM for IR) (Chen et al., 2008) to characterize the

Received March 11, 2009; revised 0ct. 27, 2009; accepted 0ct. 29, 2009.

The Wellcome Trust funded this work. C. C. Chen is funded by the National Science Council of Taiwan (TMS-094-1-A-037). We thank Peter Aston and Eric Featherstone for the design and programming involved in creating the hand grip manipulandum and Dr. Vladimir Litvak for comments on the presentation of this work.

Correspondence should be addressed to Chun-Chuan Chen, Graduate Institute of Biomedical Engineering, $\mathrm{Na}$ tional Central University, Taiwan, Jhongda Road, Jhongli City, Taoyuan County, 32001, Taiwan. E-mail c.chen@ fil.ion. ucl.ac.uk.

DOI:10.1523/JNEUROSCI.1194-09.2010

Copyright $\odot 2010$ the authors $\quad 0270-6474 / 10 / 298393-07 \$ 15.00 / 0$ modulation of frequency-dependent coupling among neuronal sources during action execution.

Hand movements have been shown to modulate oscillatory power at different frequencies, such as alpha event-related desynchronization (ERD) and beta event-related synchronization (ERS) in brain areas engaged by action execution; e.g., primary motor cortex (M1), supplementary motor area (SMA) and premotor areas (PM) (Pfurtscheller and Andrew, 1999). Movementrelated modulations in power have been seen consistently across different spatial scales, from single unit recordings, through local field potential recordings, to macroscopic measures such as electroencephalogram (EEG) or magnetoencephalogram (MEG) (Leocani et al., 1997; Crone et al., 1998a,b; Kilner et al., 2003). Given that action execution induces changes in different frequencies within connected brain regions, we wanted to model how these oscillations are orchestrated during motor control. The nature of this frequency-specific coupling is important, given that excessive synchronization at distinct frequencies is seen in some pathological conditions. For example, in Parkinson's patients, synchronization at $4 \sim 6 \mathrm{~Hz}$ between the contralateral primary motor cortex and forearm muscles is thought to contribute to resting tremors, while excessive synchrony at $10-35 \mathrm{~Hz}$ in basal ganglia/subthalamic nucleus is associated with bradykinesia (for review, see Brown, 2007). The motivation for the work reported here was to establish a reference point for future clinical studies, using normal subjects and a simple established paradigm. This normative reference might help pinpoint where abnormal modulations of specific frequencies arise.

The aim of this study was to model modulations of frequencyspecific oscillations in the motor network induced by an established 
handgrip task (Ward et al., 2008). We modeled these modulations in terms of coupling between electromagnetic sources, where power in one source causes changes in the power expressed in others. Critically, we distinguished between within-frequency linear coupling and between-frequency nonlinear coupling. Specifically, we asked whether there is a difference in the relative contribution of linear and nonlinear mechanisms between intrinsic and extrinsic coupling. Our results suggest that extrinsic connectivity is best characterized as nonlinear coupling, whereas intrinsic connections are best modeled with linear coupling.

\section{Materials and Methods}

Nine healthy, right-handed subjects $(20 \sim 32$ years of age, 5 female) were recruited. Written consent was obtained from all subjects, in accordance with the Declaration of Helsinki. The study was approved by the Joint Ethics Committee of the Institute of Neurology, University College London (UCL) and National Hospital for Neurology and Neurosurgery, UCL Hospitals National Health Service Foundation Trust, London.

Subjects were instructed to perform a visually cued ballistic isometric grip, using their dominant hand with an intertrial interval of $7 \pm 2$ s. Before scanning, subjects were asked to grip the manipulandum to generate a maximum voluntary contraction (MVC). The tar-

get force was set at $45 \%$ of MVC. Subjects were trained to approximate the target force with visual feedback before scanning. However, no visual feedback was provided during scanning, to minimize activity in occipital and parietal sources. Force output was recorded using a MEGcompatible gripper and used to identify the movement onset (i.e., the reaction time, from the onset of the visual cue until the onset of the ballistic grip), the grip duration and force level.

MEG signals were measured continuously at $240 \mathrm{~Hz}$ during task performance using a whole-head CTF Omega $275 \mathrm{MEG}$ system. At the beginning and end of each measurement, the positions of three anatomical landmarks (bilateral preauricular points and nasion) were recorded to exclude excessive head movement (thresholded at $1.5 \mathrm{~cm}$ and the measured maximal translation across subjects $<1.3 \mathrm{~cm} ; 2.68 \sim 12.68 \mathrm{~mm}$ ).

The MEG data were preprocessed offline using SPM8 (SPM8, Wellcome Trust Centre for Neuroimaging, http://www.fil.ion.ucl.ac.uk/ $\mathrm{spm} /$ ). The data were epoched from -500 to $+1000 \mathrm{~ms}$, where time 0 indicates movement onset. Poorly performed (reaction times of more than one sec) and artifact contaminated (MEG amplitude $>500 \mathrm{fT}$ ) trials were excluded from further analysis; resulting in 88-98 artifact-free epochs (889890989496909395) with 642.66 \pm 54.92 ms mean reaction time and $639.45 \pm 54.48 \mathrm{~ms}$ grip duration. The mean force level was $45 \pm$ $25 \%$ of subject-specific MVC. These artifact-free epochs were projected from channel space to the sources using the generalized inverse of the lead-field matrix for our chosen sources (see Model specification below). The spectral density from 4 to $48 \mathrm{~Hz}$ at each source was computed over peristimulus time using a time-frequency Morlet wavelet transform (wavelet number: 7). The frequency ranges cover the theta $(4-8 \mathrm{~Hz})$, alpha $(8-15 \mathrm{~Hz})$, beta $(15-30 \mathrm{~Hz})$, and gamma $(>30 \mathrm{~Hz})$ bands. The absolute values of the resulting time-frequency responses were averaged over trials and baseline-corrected by subtracting the frequency-specific power of the first time-bin. For computational expediency, we reduced the dimensionality of spectra into four principal frequency components derived from a singular value decomposition of the spectra so that the extracted frequency modes are subject-specific. This procedure accounts for the huge interindividual variability of engaged frequencies in motor system (Kilner et al., 2000; Omlor et al., 2007), particularly within the alpha band (Pfurtscheller and Lopes da Silva, 1999). Those singular components preserve $>93 \%$ of the spectral variance in all subjects (range $93 \% \sim 97 \%$ ) of which $68.97 \%$ (ranged $56.03 \% \sim 78.70 \%$ ) of total energy was contributed by the low-frequency components $(\leq 20 \mathrm{~Hz})$. The resulting spectral dynamics enter DCM as the observations that the model is trying to explain.

DCM specification (sources and coupling). The source locations were taken from the group results of a functional magnetic resonance imaging (fMRI) study using the identical task, where five subjects performed 25 ballistic isometric hand grips to $45 \%$ of MVC. Imaging data were analyzed using SPM8 as described previously (Ward et al., 2008). The localizations were taken as the peak coordinate in Montreal Neurological Institute (MNI) space within each significant cluster (voxels significant at $p<0.05$, corrected for multiple comparisons across the whole brain). Peak increases in activity were seen in left primary motor cortex (M1), bilateral premotor cortices (PM) and left supplementary motor area (SMA). In addition, right M1 was included because of significant taskrelated deactivation during hand grip secondary to transcallosal interhemispheric inhibition (Ward et al., 2008). Using these five sources we then specified 12 different connectivity models as shown in Figure 1.

This model space was constructed using three model attributes. First, whether intrinsic (I) connections are linear (L) or nonlinear $(\mathrm{N})$ and second, whether extrinsic (E) connections are linear or nonlinear. This results in four sorts of models. The third attribute was the lateralization of cross-hemispheric coupling between PM and M1. We modeled three levels of this lateralization: right PM to left M1 (r-), or left PM to right M1 (1-) or both (b-). This gave a total of 12 models, as shown in Figure 1 . We use the designation r-ILEL to mean a right-lateralized architecture, where intrinsic connections are linear and extrinsic connections are linear.

Dynamic causal modeling for induced responses. Dynamic causal modeling (DCM) entails the use of dynamic or state-space models to explain observed time-series of data features (Friston et al., 2003; David et al., 


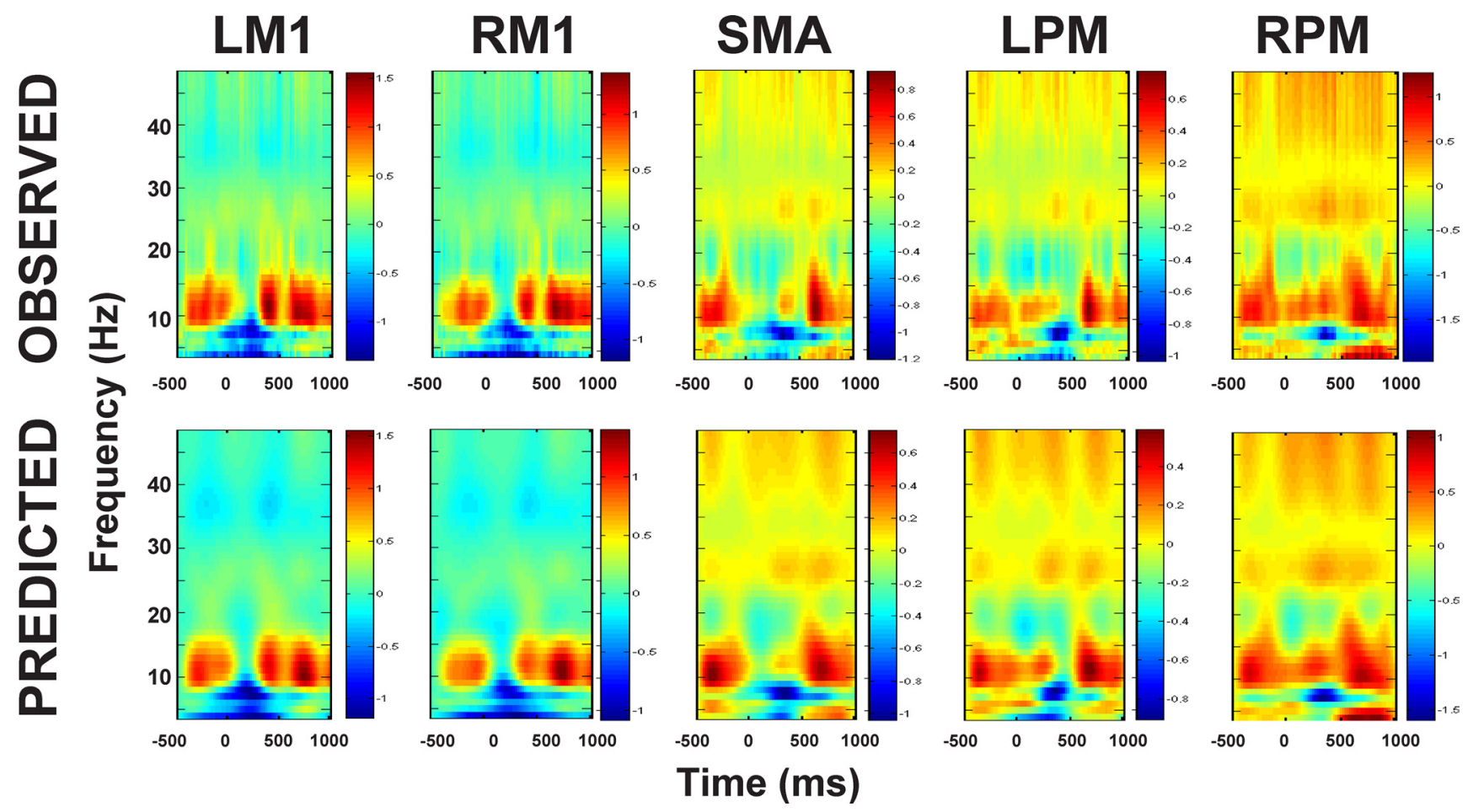

Figure 2. The observed (top) and predicted (bottom) spectral responses for a representative subject under the best mode (I-ILEN) from the Bayesian model comparison.

2006). Recently, we described a dynamic causal model for spectral responses as measured by EEG or MEG (Chen et al., 2008) and have used it to assess nonlinear coupling among visual areas during face processing (Chen et al., 2009). In brief, DCM for IR enables the user to model event-related spectral responses, over a range of frequencies, as the response of a distributed set of coupled electromagnetic sources to a spectral perturbation. The model parameters encode the frequency response of each source to exogenous input and the coupling among sources and different frequencies. Bayesian inversion of these models allows one to compare different models or hypotheses and enables inferences about the parameters of the best model.

Inference on models: Bayesian model comparison. At a single subject level, we compared the difference in log model evidence or marginal likelihoods between models, i.e., log Bayes factor (Penny et al., 2004) to identify the best among the models tested. To identify the model with the most evidence at the group level we added the log-evidences from each subject, under the assumption that each subject's data are conditionally independent of each other. To ensure differences in log-evidence were consistent across subjects, the log-evidences for each model, over the nine subjects, were entered into a repeated-measures ANOVA with three within-subject factors (distance with two levels, nonlinearity with two levels, and laterality with three levels-corresponding to the columns and rows of Fig. 1, respectively).

Model parameters: Visualization of coupling matrices. To quantify the coupling under the best model, for each intrinsic or extrinsic connection the (frequency-to-frequency) matrices of subject-specific estimates were smoothed (to account for intersubject variability in frequency-tofrequency coupling using a Gaussian kernel with full-width halfmaximum of $8 \mathrm{~Hz}$ ). These were then averaged by entering them into a conventional SPM analysis to identify reliable frequency-specific connectivity. We report the average coupling strengths in, and only in, nonzero frequency bins (at $p<0.005$ uncorrected).

\section{Results}

Time-frequency responses at source level

The estimated event-related spectral responses at the source level of a representative subject are shown in Figure 2 (top), where the alpha power decreases bilaterally in M1 from movement onset to
$300 \mathrm{~ms}$. This pattern was observed consistently across all subjects. Transient beta power increases were seen in bilateral M1, SMA and left PM (6 of 9 subjects), and enhanced gamma was seen in SMA and PM bilaterally (8 of 9). We also observed transient bilaterally M1 beta power decreases in some subjects (5 of 9). Together, these time-frequency responses are in line with previous findings (Leocani et al., 1997; Crone et al., 1998a,b; Kilner et al., 2004). The bottom in Figure 2 shows the predicted spectral responses, at the source level, by the best (l-ILEN) model (see Inference on models for details). Note that the spectra are normalized individually with respect to their maximum. It is clear that the model can explain the data very well. On average, the 1-ILEN model explains $94.17 \%$ data variance (range $86.78 \sim 98.01 \%$ ).

\section{Inference on models: Nonlinear effect and motor networks}

Figure 3 summarizes the results of our model comparison. It is immediately obvious that the models which fit the data best are those with nonlinear extrinsic coupling (Fig. 3A). Models with one or more nonlinear connection were consistently better than purely linear models in every subject (Fig. 3B) and an ILEN model was the best in six of nine subjects. In addition, model comparison also clearly shows strong evidence for model ILEN, summed over the factors of lateralization and subjects (assuming all subjects expressed the same unknown model; see Stephan et al., 2009 and Fig. 3C). This is supplemental evidence that shows we can differentiate the nonlinear intrinsic coupling from linear coupling, when the extrinsic coupling is nonlinear. The l-ILEN model was the best model among those tested at the group level, with a Bayes factor of 1857 (l-ILEN vs r-INEN; i.e., the best vs the next best). Bayes factor measures the marginal likelihood ratio of two models using their model evidence and usually a Bayes factor of approximately 20 is considered as "strong" evidence in favor of one model relative to another (Penny et al., 2004). It is important to note that the most complex model (b-INEN) is not necessarily 
the best (only one subject had this model as the best). This is because the logevidence includes a penalty term for complexity (see Friston et al., 2003 and Penny et al., 2004 for details). The repeatedmeasure ANOVA confirms our BMS result that there is strong evidence for nonlinearity $\left(F_{(1,7)}=15.355, p=0.006\right)$, distance $\left(F_{(1,7)}=19.539, p=0.003\right)$ and their interaction $\left(F_{(1,7)}=15.615, p=0.006\right)$ in terms of consistency over subjects. This mirrors the BMS conclusion that extrinsic connectivity was nonlinear while intrinsic connectivity was essentially linear. There was no effect of laterality (levels: right, bilateral and left lateralized models; $F_{(1.13,9.14)}=0.951$, $p=0.381)$ nor any interaction with the other two factors.

In summary, in normal subjects we found very strong evidence for nonlinear coupling between areas and linear interactions between areas. Note that our conclusion about intrinsic linear coupling is based on the fact that there was no consistent evidence for intrinsic nonlinear coupling. This lack of evidence might suggest that our data were simply insufficient to reveal a small nonlinear contribution within area; however, the clear evidence for nonlinear effects in the extrinsic connections suggests that, at least quantitatively, nonlinearities predominate in extrinsic, relative to intrinsic interactions. In addition, there is evidence for an asymmetry of interhemispheric interactions in the right hand movement task we used; although this was less consistent over subjects.

\section{Coupling parameters}

Statistical tests are repeated for each element of the coupling parameter matrices from all subjects, under the l-ILEN model. Each of these matrices represents the frequency-to-frequency coupling associated with one connection. The corresponding SPMs of the T-statistic (thresholded at $p<0.005$ uncorrected) are shown for "excitatory" (positive; Fig. $4 A$ ) and "inhibitory" (negative; Fig. $4 B$ ) effects respectively. As seen in Figure 4, we found several instances of consistent nonlinear interactions. A summary of these $t$ test results is provided in Table 1. These seem to be more profound when the coupling is negative. For instance, several consistent regions of negative coupling are found far from the (within-frequency), leading diagonal compared with the positive coupling SPMs. When considering reciprocal connections, the frequencies entailed by nonlinear coupling appear asymmetric. For example, in Figure $4 B$ (arrows), the negative coupling from LPM to RM1 involves alpha-gamma coupling, while the reciprocal RM1 to LPM connection shows significant gamma-theta coupling. In the same vein, theta oscillations in SMA suppress gamma oscillations in LM1 but, from LM1 to SMA, the negative coupling was between gamma and alpha. The more quantitative characterizations of the nonlinear coupling identified by Bayesian model comparison speak to the complicated nature of nonlinear interactions in the brain, even when modeled as simply as with DCM for induced responses.

\section{Log-evidence}
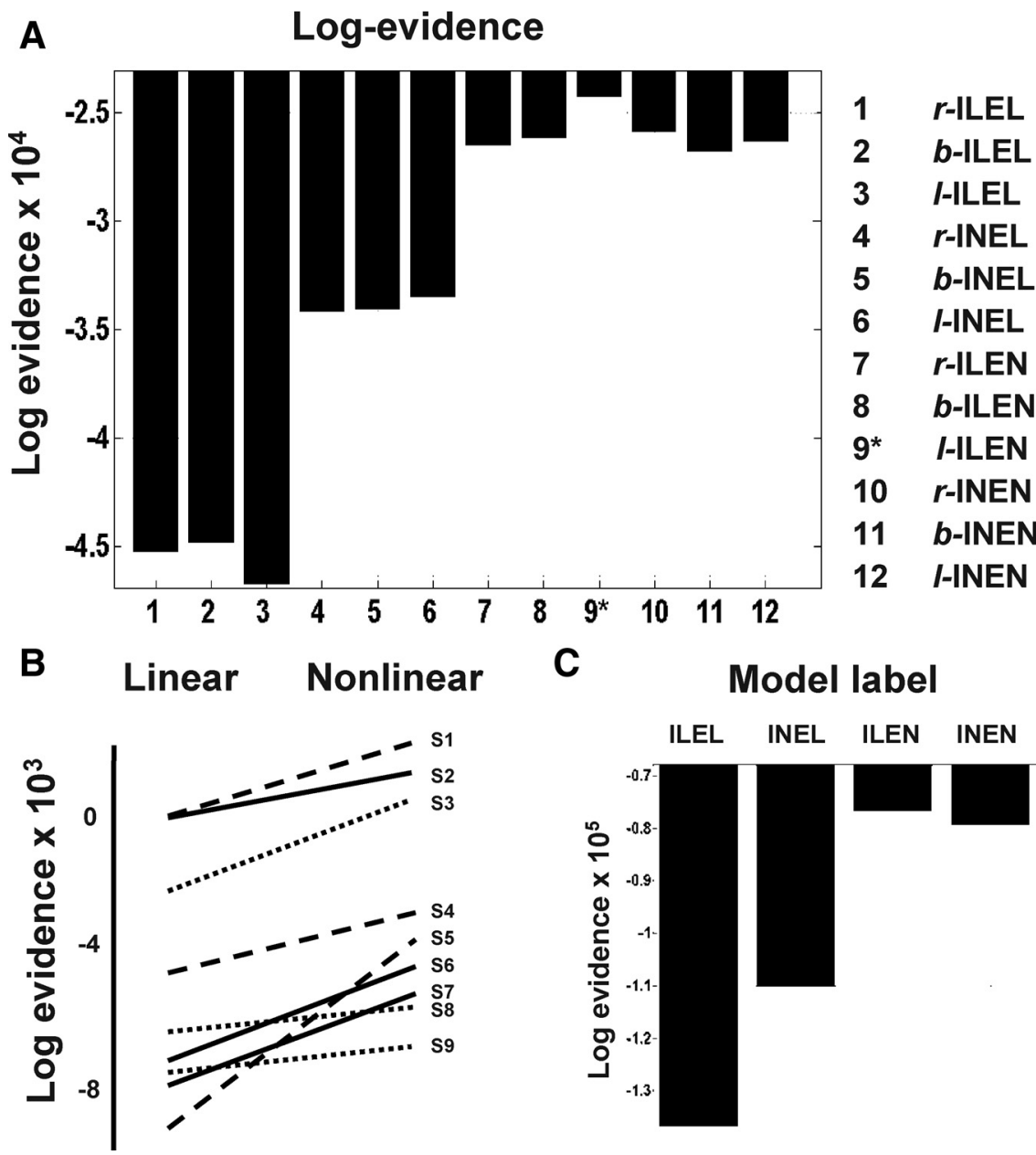

Figure 3. Results of Bayesian model selection (BMS). $A$, Pooled log-evidences of the $12 \mathrm{DCM}$ tested. It can be seen that the best model is I-ILEN (log-evidence $=-24,297$ ) and the next best is $\boldsymbol{b}$-ILEN (log-evidence $=-25,880) . \boldsymbol{B}$, Comparison of the average slopes over all subjects indicate that the nonlinear coupling is essential as the nonlinear models are always better than the linear ones. C, BMS on partitioned model space reveals that the ILEN model is the best model.

Note that the significance of the negative linear intrinsic coupling in Figure $4 B$ is due largely to prior constraints on the parameters. We use these priors to ensure the system is dissipative (see Friston et al., 2003 for discussion of this constraint). In other words, the system will decay to a stable fixed point (equilibrium point) after a period of time. Given that we know the rough time-constants of induced electromagnetic responses, we can use this information to place a prior expectation on the decay rates, following experimental perturbation. This decay is implemented by intrinsic (self) inhibition (cf. Friston et al., 2003).

\section{Discussion}

In this study, we provide empirical evidence for nonlinear coupling among distributed neuronal sources in the motor system and that these play an important role in modulating spectral responses under normal conditions. Interestingly, we found no evidence for nonlinear or between-frequency coupling intrinsic to each source, suggesting that linear or driving mechanisms may be a sufficient account of interactions among local neuronal populations. In addition, we found evidence for an asymmetric interhemispheric interaction involving right PM in our right-hand movement task. A quantitative examination of the extrinsic or long-range coupling parameters, showed some interesting asym- 


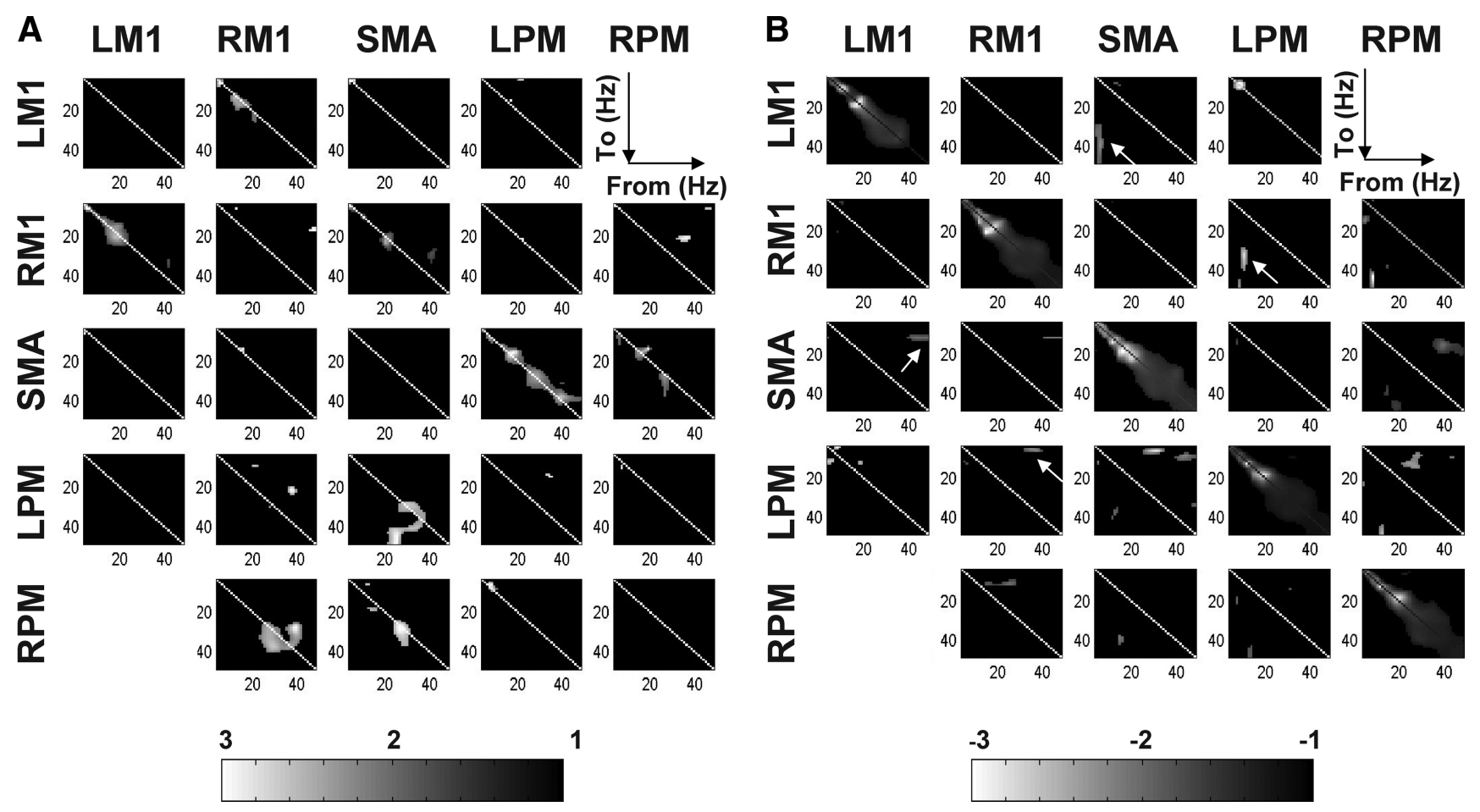

Figure 4. Statistical results of coupling parameters. $A, S P M\{P\}$ of the positive coupling strengths (one-tailed), thresholded at $p<0.005$ uncorrected. This shows regions of frequency-frequency space, where the average coupling was great than zero. $\boldsymbol{B}$, SPM $\{\mathcal{F}\}$ of negative coupling parameters. The arrows indicate functional asymmetries in terms of frequency-specific coupling. The significance of the linear intrinsic coupling is largely due to prior constraints on the parameters (see Results for details).

Table 1. Summary of the SPM analysis of the coupling parameters

\begin{tabular}{|c|c|c|c|c|c|}
\hline & LM1 & RM1 & SMA & LPM & RPM \\
\hline LM1 & & $\begin{array}{l}\text { theta-theta }(+) \\
\text { alpha/beta-beta }(+)\end{array}$ & $\begin{array}{l}\text { theta-theta }(+) \\
\text { theta-gamma }(-) \\
\text { alpha-alpha }(-)\end{array}$ & $\begin{array}{l}\text { beta-theta }(+) \\
\text { alpha-alpha }(-)\end{array}$ & \\
\hline RM1 & $\begin{array}{l}\text { beta-beta }(+) \\
\text { gamma-gamma }(+)\end{array}$ & & $\begin{array}{l}\text { beta-beta }(+) \\
\text { gamma-gamma }(+)\end{array}$ & gamma-alpha (-) & $\begin{array}{l}\text { gamma-beta }(+) \\
\text { gamma-theta }(+) \\
\text { theta-alpha }(-) \\
\text { alpha-gamma }(-)\end{array}$ \\
\hline SMA & gamma-alpha (-) & $\begin{array}{l}\text { alpha-alpha }(+) \\
\text { gamma-alpha }(-)\end{array}$ & & $\begin{array}{l}\text { alpha-alpha }(+) \\
\text { beta-beta }(+) \\
\text { gamma-gamma (+) } \\
\text { alpha-alpha (-) }\end{array}$ & $\begin{array}{l}\text { theta-alpha }(+) \\
\text { beta-beta }(+) \\
\text { gamma-gamma (+) } \\
\text { beta-gamma (-) } \\
\text { gamma-beta (-) }\end{array}$ \\
\hline LPM & $\begin{array}{l}\text { theta-alpha }(-) \\
\text { alpha-theta }(-) \\
\text { beta-alpha }(-)\end{array}$ & $\begin{array}{l}\text { beta-alpha }(+) \\
\text { gamma-beta }(+) \\
\text { theta-alpha }(-) \\
\text { gamma-theta }(-)\end{array}$ & $\begin{array}{l}\text { beta-gamma }(+) \\
\text { gamma-gamma }(+) \\
\text { theta-alpha }(-) \\
\text { alpha-gamma }(-) \\
\text { gamma-theta/alpha }(-) \\
\text { gamma-gamma }(-)\end{array}$ & & $\begin{array}{l}\text { alpha-gamma (-) } \\
\text { beta-alpha (-) }\end{array}$ \\
\hline RPM & & $\begin{array}{l}\text { gamma-gamma }(+) \\
\text { beta-alpha }(-)\end{array}$ & $\begin{array}{l}\text { alpha-theta }(+) \\
\text { alpha-beta }(+) \\
\text { gamma-gamma }(+) \\
\text { beta-gamma }(-)\end{array}$ & $\begin{array}{l}\text { alpha-alpha }(+) \\
\text { alpha-beta }(-) \\
\text { beta-gamma }(-) \\
\text { gamma-alpha }(-)\end{array}$ & \\
\hline
\end{tabular}

+ denotes positive coupling and - denotes negative coupling.

metries in frequency space and that this coupling was predominantly negative or suppressive.

Intrinsic (local) linear and extrinsic (global) nonlinear effects A recent pharmacological study in rat brains has shown that coapplication of kainic acid and carbachol to layer V in M1 can reliably induce synchronous oscillatory activity in the beta frequency band in layer II to VI (Yamawaki et al., 2008). These results imply that interlaminar influences may be mediated by driving or linear effects because they induce distributed oscillations at the same frequency. Furthermore, it has been shown that in pathological conditions such as Parkinsonism, abnormal oscillatory synchronization of neuronal populations in cortex, subthalamic nucleus and basal ganglia can lead to movement impairment (Levy et al., 2002; Priori et al., 2004; Marceglia et al., 2006; Brown, 2007). Our findings suggest that the local interac- 
tions may be predominantly linear under normal conditions. Other studies have demonstrated nonlinear coupling in EEG/ MEG signals in a variety of tasks, systems and pathological conditions (Breakspear, 2002; Kotini and Anninos, 2002; Chávez et al., 2003) leading to suggestions that nonlinear coupling is an important aspect of functional integration (Tallon-Baudry and Bertrand, 1999; Varela et al., 2001; Jensen and Colgin, 2007; Chen et al., 2009). We have shown that during the performance of a simple motor task both linear and nonlinear coupling is likely to be present. Specifically, our results demonstrate that local interactions can be explained by linear coupling, but that coupling between regions is nonlinear in nature.

In terms of cross-frequency coupling, bicoherence and nested oscillations are other important expressions of nonlinear coupling that may reflect key physiological mechanisms of functional integration and dynamic coordination in the brain. Bicoherence has been intensively used as an index of anesthesia (Hayashi et al., 2008) while nested oscillations are observed largely during memory tasks, such as theta-gamma, theta-beta and theta-gamma, theta-beta/gamma coupling (Lisman and Idiart, 1995) and during sleep [infra-slow oscillations (ISOs): $0.02 \sim 0.2-1 \mathrm{~Hz}$ ] (Vanhatalo et al., 2004) and even during the resting state:alpha-high gamma frequencies. However, we are not aware of any applications within the central motor system using these methods. Our results suggest that nonlinearities in coupling are expressed predominantly in extrinsic connections. This may reflect the fact afferent inputs from other regions exert nonlinear effects on intrinsic dynamics. This nonlinearity may be at the level of postsynaptic receptors (e.g., NMDA receptors that are preferentially targeted by extrinsic backward connections in the brain); or at the level of lumped time-constants governing intrinsic population dynamics.

\section{Asymmetry of interhemispheric connections}

Asymmetry in the human brain architectures has been shown in many studies, both functionally or anatomically (Rockland and Pandya, 1979; Zeki and Shipp, 1988). In the motor system, handedness and experience-dependent plasticity are thought to be the main factors subtending these asymmetric properties (Karni et al., 1995; Haaland et al., 2000; Klöppel et al., 2007). Moreover, functional lateralization of motor control can be altered by pathological or traumatic changes (Ward and Cohen, 2004). In this study, we were able to quantify task-dependent frequencyspecific causal influences mediating the observed spectral responses and characterize functional asymmetry in terms of long-range coupling. Bayesian model comparison suggested that the left lateralized ILEN model was superior to the symmetric homolog. Our left-lateralized model conforms to the left hemisphere dominance, expressed for example in the asymmetric engagement of premotor cortex during skilled movement in right-handed adults (Pollok et al., 2006).

\section{Asymmetry of hierarchical connections}

In addition to hemispheric asymmetries, frequency-specific coupling was distinct in forward and backward connections, especially between the SMA and premotor sources. Furthermore, predominant positive and negative couplings are located in different frequency bands in most connections. For example, the gamma rhythm in left M1 inhibits the alpha activity in SMA but no consistent positive connection was found.

It is important to establish the normal pattern of the frequency-specific interactions in the motor system because several movement disorders show frequency-related abnormalities, such as resting tremors $(4 \sim 6 \mathrm{~Hz})$ and bradykinesia $(10-35 \mathrm{~Hz})$ (Brown, 2007). However, the details of the underlying mechanisms remain largely unknown. Our study provides a qualitative and quantitative characterization of frequency-specific effects under normal conditions, which we hope will be useful when studying induced responses in patients. To our knowledge, this is the first study of frequency-specific coupling in the motor network under normal conditions. Given that, in the motor system, induced responses depend on the task and show substantial between-subject variability (Kilner et al., 2000; Kristeva et al., 2007; Omlor et al., 2007), we do not anticipate out results will generalize to other movement-related networks. Rather, we consider our study as a reference point for similar studies in patients using the same paradigm.

\section{The question of model specification}

It is important to note that any DCM analysis is conditioned on the models considered. For example, our conclusions are specific to the particular sources that we chose to model the data. Specifically, we mapped the observed MEG data into source space by multiplying the generalized inverse lead field matrix given the source locations (see Chen et al., 2008 for details). This generalized inverse of the lead-field is one of many inversion schemes that can be used to project data from channel to source space (Michel et al., 2004; Friston et al., 2008). The advantage of this projection is that there is a unique solution for the data features, given the prior specification of source locations. This approach leads to a source spectrum, which is a reasonable summary of the real neuronal sources generating the data. Therefore, if any sources are omitted or misplaced, there might be a better model of the data and possibly a different conclusion from model comparison. If one did not know where the spectral signals were generated, the beam-former method could be one useful strategy that allows one to localize the source positions and estimate spectral features empirically (Sekihara et al., 2006). Once these locations have been established, the generalized inverse of the associated lead-field matrix furnishes a near-optimum equivalent current dipoles (ECD) summary of activity that avoids suppression of local correlated activity. In this study, we adopted fMRI results as our prior on source locations. This is based on the fact that there is a congruity between fMRI activity and the corresponding MEG dipoles in sensorimotor cortex (Korvenoja et al., 2006). The sensitivity of our conclusions to the particular sources chosen may not be very severe for two reasons. First, there is little localizing information in electromagnetic signals. This means that if we had changed the source locations, the data features would not change very much (this is because we pool spectral estimates over all orientations and the orientation of the implicit dipole is essentially free). Second, including more sources would not affect the conclusions about effective connectivity; in the sense that effective connectivity can be polysynaptic and could be mediated by intervening (unmodeled) sources. The only worry here is that choosing the wrong number of sources leaves variance in the data-features unexplained (or wrongly attributed). This is unlikely, given the high proportion of variance explained by the sources we used.

In conclusion, we have established the prevalence of nonlinear or between-frequency coupling among distributed components of the motor system during a simple motor task. These extrinsic nonlinear interactions appear to unfold in the context of local or intrinsic linear coupling within each area. The associated taskdependent motor network has asymmetric features, as reflected in both the deployment of connections and the frequency speci- 
ficity of reciprocal connections. In a future paper, we will show how this motor network is affected by healthy aging and by corticospinal system damage secondary to stroke.

\section{References}

Andrew C, Pfurtscheller G (1996) Event-related coherence as a tool for studying dynamic interaction of brain regions. Electroencephalogr Clin Neurophysiol 98:144-148.

Breakspear M (2002) Nonlinear phase desynchronization in human electroencephalographic data. Hum Brain Mapp 15:175-198.

Brown P (2007) Abnormal oscillatory synchronisation in the motor system leads to impaired movement. Curr Opin Neurobiol 17:656-664.

Chávez M, Martinerie J, Le Van Quyen M (2003) Statistical assessment of nonlinear causality: application to epileptic EEG signals. J Neurosci Methods 124:113-128.

Chen CC, Kiebel SJ, Friston KJ (2008) Dynamic causal modelling of induced responses. Neuroimage 41:1293-1312.

Chen CC, Henson RN, Stephan KE, Kilner JM, Friston KJ (2009) Forward and backward connections in the brain: a DCM study of functional asymmetries. Neuroimage 45:453-462.

Crone NE, Miglioretti DL, Gordon B, Sieracki JM, Wilson MT, Uematsu S, Lesser RP (1998a) Functional mapping of human sensorimotor cortex with electrocorticographic spectral analysis. I. Alpha and beta eventrelated desynchronization. Brain 121:2271-2299.

Crone NE, Miglioretti DL, Gordon B, Lesser RP (1998b) Functional mapping of human sensorimotor cortex with electrocorticographic spectral analysis. II. Event-related synchronization in the gamma band. Brain 121:2301-2315.

David O, Kiebel S, Harrison LM, Mattout J, Kilner JM, Friston KJ (2006) Dynamic causal modelling of evoked responses in EEG and MEG. Neuroimage 20:1255-1272.

Friston KJ, Harrison L, Penny W (2003) Dynamic causal modelling. Neuroimage 19:1273-1302.

Friston K, Harrison L, Daunizeau J, Kiebel S, Phillips C, Trujillo-Barreto N, Henson R, Flandin G, Mattout J (2008) Multiple sparse priors for the M/EEG inverse problem. Neuroimage 39:1104-1120.

Gerloff C, Richard J, Hadley J, Schulman AE, Honda M, Hallett M (1998) Functional coupling and regional activation of human cortical motor areas during simple, internally paced and externally paced finger movements. Brain 121:1513-1531.

Gross J, Kujala J, Hamalainen M, Timmermann L, Schnitzler A, Salmelin R (2001) Dynamic imaging of coherent sources: studying neural interactions in the human brain. Proc Natl Acad Sci U S A 98:694-699.

Haaland KY, Harrington DL, Knight RT (2000) Neural representations of skilled movement. Brain 123:2306-2313.

Hayashi K, Sawa T, Matsuura M (2008) Anesthesia depth-dependent features of electroencephalographic bioherence spectrum during sevoflurane anesthesia. Anesthesiology 108:841-850.

Jensen O, Colgin LL (2007) Cross-frequency coupling between neuronal oscillations. Trends Cogn Sci 11:267-269.

Kahana MJ, Caplan JB, Sekuler R, Madsen JR (1999) Using intracranial recordings to study theta. Response to J. O'Keefe and N. Burgess (1999). Trends Cogn Sci 3:406-407.

Karni A, Meyer G, Jezzard P, Adams MM, Turner R, Ungerleider LG (1995) Functional MRI evidence for adult motor cortex plasticity during motor skill learning. Nature 377:155-158.

Kilner JM, Baker SN, Salenius S, Hari R, Lemon RN (2000) Human cortical muscle coherence is directly related to specific motor parameters. J Neurosci 20:8838-8845.

Kilner JM, Salenius S, Baker SN, Jackson A, Hari R, Lemon RN (2003) Taskdependent modulations of cortical oscillatory activity in human subjects during a bimanual precision grip task. Neuroimage 18:67-73.

Klöppel S, van Eimeren T, Glauche V, Vongerichten A, Münchau A, Frackowiak RS, Büchel C, Weiller C, Siebner HR (2007) The effect of handedness on cortical motor activation during simple bilateral movements. Neuroimage 34:274-280.

Korvenoja A, Kirveskari E, Aronen HJ, Avikainen S, Brander A, Huttunen J, Ilmoniemi RJ, Jääskeläinen JE, Kovala T, Mäkelä JP, Salli E, Seppä M (2006) Sensorimotor cortex localization: comparison of magnetoen- cephalography, functional MR imaging, and intraoperative cortical mapping. Radiology 241:213-222.

Kotini A, Anninos P (2002) Detection of non-linearity in schizophrenic patients using magnetoencephalography. Brain Topogr 15:107-113.

Leocani L, Toro C, Manganotti P, Zhuang P, Hallett M (1997) Event-related coherence and event-related desynchronization/synchronization in the $10 \mathrm{~Hz}$ and $20 \mathrm{~Hz}$ EEG during self-paced movements. Electroencephalogr Clin Neurophysiol 104:199-206.

Levy R, Ashby P, Hutchison WD, Lang AE, Lozano AM, Dostrovsky JO (2002) Dependence of subthalamic nucleus oscillations on movement and dopamine in Parkinson's disease. Brain 125:1196-1209.

Lisman J, Idiart MA (1995) Storage of $7 \pm$ short-term memories in oscillatory subcycles. Science 267:1512-1515.

Marceglia S, Foffani G, Bianchi AM, Baselli G, Tamma F, Egidi M, Priori A (2006) Dopamine-dependent non-linear correlation between subthalamic rhythms in Parkinson's disease. J Physiol 571:579-591.

Michel CM, Murray MM, Lantz G, Gonzalez S, Spinelli L, Grave de Peralta R (2004) EEG source imaging. Clin Neurophysiol 115:2195-2222.

Omlor W, Patino L, Hepp-Reymond MC, Kristeva R (2007) Gamma-range corticomuscular coherence during dynamic force output. Neuroimage 34:1191-1198.

Penny WD, Stephan KE, Mechelli A, Friston KJ (2004) Comparing dynamic causal models. Neuroimage 22:1157-1172.

Pfurtscheller G, Andrew C (1999) Event-related changes of band power and coherence: methodology and interpretation. J Clin Neurophysiol 16:512-519.

Pfurtscheller G, Lopes da Silva FH (1999) Event-related EEG/MEG synchronization and desynchronization: basic principles. Clin Neurophysiol 110: 1842-1857.

Pollok B, Gross J, Schnitzler A (2006) Asymmetry of interhemispheric interaction in left-handed subjects. Exp Brain Res 175:268-275.

Priori A, Foffani G, Pesenti A, Tamma F, Bianchi AM, Pellegrini M, Locatelli M, Moxon KA, Villani RM (2004) Rhythm-specific pharmacological modulation of subthalamic activity in Parkinson's disease. Exp Neurol 189:369-379.

Rockland KS, Pandya DN (1979) Laminar origins and terminations of cortical connections of the occipital lobe in the rhesus monkey. Brain Res 179:3-20.

Rodriguez E, George N, Lachaux JP, Martinerie J, Renault B, Varela FJ (1999) Perception's shadow: long-distance synchronization of human brain activity. Nature 397:430-433.

Sekihara K, Hild KE 2nd, Nagarajan SS (2006) A novel adaptive beamformer for MEG source reconstruction effective when large background brain activities exist. IEEE Trans Biomed Eng 53:1755-1764.

Serrien DJ, Orth M, Evans AH, Lees AJ, Brown P (2005) Motor inhibition in patients with Gilles de la Tourette syndrome: functional activation patterns as revealed by EEG coherence. Brain 128:116-125.

Singer W, Gray CM (1995) Visual feature integration and the temporal correlation hypothesis. Annu Rev Neurosci 18:555-586.

Stephan KE, Penny WD, Daunizeau J, Moran RJ, Friston KJ (2009) Bayesian model selection for group studies. Neuroimage 46:1004-1017.

Tallon-Baudry C, Bertrand O (1999) Oscillatory gamma activity in humans and its role in object representation. Trends Cogn Sci 3:151-162.

Vanhatalo S, Palva JM, Holmes MD, Miller J, Voipio JW, Kaila K (2004) Infraslow oscillations modulate excitability and interictal epileptic activity in the human cortex during sleep. Proc Natl Acad Sci U S A 101:50535057.

Varela F, Lachaux JP, Rodriguez E, Martinerie J (2001) The brainweb: phase synchronization and large-scale integration. Nat Rev Neurosci 2:229-239.

Ward NS, Cohen LG (2004) Mechanisms underlying recovery of motor function after stroke. Arch Neurol 61:1844-1848.

Ward NS, Swayne OB, Newton JM (2008) Age-dependent changes in the neural correlates of force modulation: an fMRI study. Neurobiol Aging 29:1434-1446.

Yamawaki N, Stanford IM, Hall SD, Woodhall GL (2008) Pharmacologically induced and stimulus evoked rhythmic neuronal oscillatory activity in the primary motor cortex in vitro. Neuroscience 151:386-395.

Zeki S, Shipp S (1988) The functional logic of cortical connections. Nature 335:311-317. 